\title{
BMJ Open The Bipolar Illness Onset study: research protocol for the BIO cohort study
}

\author{
Lars Vedel Kessing, ${ }^{1,2}$ Klaus Munkholm, ${ }^{1}$ Maria Faurholt-Jepsen, ${ }^{1}$ \\ Kamilla Woznica Miskowiak, ${ }^{1,2}$ Lars Bo Nielsen, ${ }^{2,3}$ Ruth Frikke-Schmidt, ${ }^{2,3}$ \\ Claus Ekstrøm, ${ }^{4}$ Ole Winther, ${ }^{5,6}$ Bente Klarlund Pedersen, ${ }^{7}$ \\ Henrik Enghusen Poulsen, ${ }^{8}$ Roger S McIntyre, ${ }^{9}$ Flavio Kapczinski, ${ }^{10}$ \\ Wagner F Gattaz, ${ }^{11}$ Jakob Bardram, ${ }^{5}$ Mads Frost, ${ }^{12}$ Oscar Mayora, ${ }^{13}$ \\ Gitte Moos Knudsen, ${ }^{14}$ Mary Phillips, ${ }^{15}$ Maj Vinberg ${ }^{1,3}$
}

To cite: Kessing LV, Munkholm K, FaurholtJepsen $\mathrm{M}$, et al. The Bipolar IIIness Onset study: research protocol for the BIO cohort study. BMJ Open 2017;7:e015462. doi:10.1136/ bmjopen-2016-015462

- Prepublication history for this paper is available online. To view these files please visit the journal online (http://dx.doi. org/10.1136/bmjopen-2016015462).

Received 12 December 2016 Revised 19 May 2017 Accepted 19 May 2017

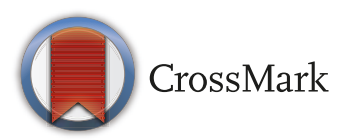

For numbered affiliations see end of article.

Correspondence to

Proffesor Lars Vedel Kessing; lars.vedel.kessing@regionh.dk

\section{ABSTRACT}

Introduction Bipolar disorder is an often disabling mental illness with a lifetime prevalence of $1 \%-2 \%$, a high risk of recurrence of manic and depressive episodes, a lifelong elevated risk of suicide and a substantial heritability. The course of illness is frequently characterised by progressive shortening of interepisode intervals with each recurrence and increasing cognitive dysfunction in a subset of individuals with this condition. Clinically, diagnostic boundaries between bipolar disorder and other psychiatric disorders such as unipolar depression are unclear although pharmacological and psychological treatment strategies differ substantially. Patients with bipolar disorder are often misdiagnosed and the mean delay between onset and diagnosis is 5-10 years. Although the risk of relapse of depression and mania is high it is for most patients impossible to predict and consequently prevent upcoming episodes in an individual tailored way. The identification of objective biomarkers can both inform bipolar disorder diagnosis and provide biological targets for the development of new and personalised treatments. Accurate diagnosis of bipolar disorder in its early stages could help prevent the long-term detrimental effects of the illness. The present Bipolar IIIness Onset study aims to identify (1) a composite blood-based biomarker, (2) a composite electronic smartphone-based biomarker and (3) a neurocognitive and neuroimaging-based signature for bipolar disorder.

Methods and analysis The study will include 300 patients with newly diagnosed/first-episode bipolar disorder, 200 of their healthy siblings or offspring and 100 healthy individuals without a family history of affective disorder. All participants will be followed longitudinally with repeated blood samples and other biological tissues, selfmonitored and automatically generated smartphone data, neuropsychological tests and a subset of the cohort with neuroimaging during a 5 to 10 -year study period.

Ethics and dissemination The study has been approved by the Local Ethical Committee (H-7-2014-007) and the data agency, Capital Region of Copenhagen (RHP2015-023), and the findings will be widely disseminated at international conferences and meetings including conferences for the International Society for Bipolar Disorders and the World Federation of Societies for
Strengths and limitations of this study

- The Bipolar IIIness Onset (BI0) study is the first study aiming to identify (1) a composite blood-based biomarker, (2) a composite electronic smartphonebased biomarker and (3) a neurocognitive signature for bipolar disorder.

- The same biomarkers will be measured longitudinally in newly diagnosed/first-episode patients with bipolar disorder and their healthy first-generation relatives.

- The BIO study will be performed by an experienced international research group, combining expertise from all areas of the study.

- Extensive initial assessment of study procedures may result in selection of participants who are intrinsically positive towards clinical research

- Confounding effects of psychotropic medication may influence results.

- Lack of randomised interventions may limit causal interpretations of the results.

Biological Psychiatry and in scientific peer-reviewed papers.

Trial registration number NCT02888262.

\section{INTRODUCTION}

\section{Biomarkers in bipolar disorders}

Bipolar disorder is a disabling mental illness with a lifetime prevalence of $1 \%-2 \%$, a high risk of recurrence of manic and depressive episodes, a lifelong elevated risk of suicide and a substantial heritability of $60 \%-80 \%$. $^{1}$ Bipolar disorder is often conceptualised as a progressive disorder with increasing risk of recurrence for every new affective episode $^{2-5}$ and with increasing cognitive disabilities during the course of illness. ${ }^{6-9}$ Clinically, diagnostic boundaries between bipolar disorder and other psychiatric 
disorders such as unipolar disorder are unclear although some pharmacological and psychological treatment strategies differ substantially. Patients with bipolar disorder are often misdiagnosed as having unipolar disorder, transient psychosis, reaction to stress/adjustment disorder or psychoactive substance abuse, ${ }^{10}$ and the mean delay between onset and diagnosis is 5-10 years. ${ }^{11}$ Although the risk of relapse of depression and mania is high, it is for most patients impossible to predict and consequently prevent upcoming episodes in an individual tailored way. The identification of objective biomarkers as measures of pathophysiological processes can both inform bipolar disorder diagnosis and provide biological targets for the development of new and personalised treatments. ${ }^{12}$ Accurate diagnosis of bipolar disorder in its early stages could help prevent the long-term detrimental effects of the illness.

Recently, promising results have been presented regarding a diagnostic test for unipolar depression comprising levels of nine biomarkers in peripheral blood. ${ }^{13}$ Although the nature of bipolar disorder seems more biologically driven than the nature of major depression with a higher heritability, there has been no or few attempts to identify a similar composite biomarker for bipolar disorder.

\section{Onset of illness and staging in bipolar disorder}

Although the course of illness is heterogeneous, there is a body of evidence for clinical progression on average of unipolar and bipolar disorders as increasing number of affective episodes seem associated with (1) increasing risk of recurrence, (2) increasing duration of episodes, (3) increasing symptomatic severity of episodes, (4) decreasing threshold for developing episodes and (5) increasing risk of developing dementia. ${ }^{9}$ It is likely that this clinical progression with deteriorating effects of affective episodes and duration of illness is associated with neurobiological changes over the course of illness. Unfortunately, results of all longitudinal studies on the biology of bipolar disorder are hitherto hampered by three major limitations: (1) Only few studies have recruited patients with bipolar illness from onset of the illness and most of these have used first-onset mania as inclusion criteria thereby excluding patients with a hypomanic episode (bipolar disorder, type II). As the biology of bipolar disorderbased on cross-sectional studies-seems to change over the course of illness from first episode to first relapse and recurrent relapses to an unremitting or rapid cycling course, ${ }^{14-18}$ this is a major limitation in our knowledge internationally, (2) the number of patients included in prior studies is less than 200 , which is a limitation taking the heterogeneity of bipolar disorder into account (eg, bipolar disorder types I and II may have different biology, and so on), and (3) the prospective follow-up period is less than a few years in all studies.

In the Bipolar Illness Onset (BIO) study, we will establish a large cohort compromising three subcohorts that will be followed long term with systematic diagnostic, blood-based biomarkers, smartphone data and cognitive and brain imaging assessment. The three subcohorts will consist of: (1) patients with newly diagnosed/first-episode bipolar disorder and (2) their healthy first-generation siblings and offspring and (3) healthy individuals without a family history of affective disorder.

\section{Overall aims}

1. To identify a composite blood-based biomarker measure as well as a composite electronic smartphonebased biomarker from onset of bipolar disorder during progression and in later stages.

2. To investigate if the composite blood-based biomarker measure and electronic smartphone-based biomarker identified among patients with bipolar disorder predict onset of illness (depression or mania) among these patients' healthy first-generation siblings and offspring.

3. By applying an integrated systems approach, to identify patterns of 'cerebral signatures' across neurocircuitry and cognitive levels and to validate the composite blood-based biomarker and electronic smartphonebased biomarkers against these biosignatures.

4. To investigate long-term developmental trajectories in neurocognitive function and brain imaging from the high-risk state to onset of bipolar disorder following first relapse and recurrent relapses and in the late stage with an unremitting, multiepisode or rapid cycling course.

5. To investigate whether the course of illness is progressive on average in bipolar disorder and to identify corresponding changes in biomarkers during the course of illness within BIO-1 to BIO-4.

\section{METHODS AND ANALYSIS \\ Overall methods}

The BIO study is a long-term cohort study that started on April 2015 and planned to include 300 newly diagnosed/first-episode patients with bipolar disorder from the Copenhagen Affective Disorder Clinic, 200 of these patients' healthy first-generation relatives and 100 healthy individuals without a first-generation family history of affective disorders.

\section{The Copenhagen Affective Disorder Clinic}

The Copenhagen Affective Disorder Clinic is a mood disorder clinic that provides treatment service for patients with newly diagnosed/first-episode bipolar disorder ${ }^{19}$ The Copenhagen Affective Disorder Clinic receives patients from the entire Capital Region of Denmark covering a catchment area of 1.6 million people and all psychiatric centres in the region. All patients referred to the Clinic as newly diagnosed/first-episode patients, that is, onset of first manic or hypomanic episode or when the ICD-10 (International Statistical Classification of Diseases and Related Health Problems 10th Revision) diagnosis of bipolar disorder is made for the first time, will routinely 
be asked for inclusion in the BIO study. Nearly all patients treated in the Clinic have a diagnosis of bipolar disorder type I or type II whereas patients with bipolar disorder-not otherwise specified or patients with cyclothymia are not treated in the Clinic and consequently not included in the BIO study.

\section{Recruitment of the three cohorts}

1. Three hundred patients (aged 15-70 years) referred to the Copenhagen Affective Disorder Clinic as newly diagnosed/first-episode patients with bipolar disorder, that is, onset of first manic or hypomanic episode or when the diagnosis of bipolar disorder is made for the first time. The Clinic receives more than 100 newly diagnosed/first-episode patients with bipolar disorder each year and we expect that nearly all will accept participation in the BIO study as this implies an extensive clinical evaluation.

2. Two hundred first-generation relatives (siblings and children aged 15-40 years) to the recruited newly diagnosed/first-episode patients with bipolar disorder.

3. One hundred age and gender-matched healthy individuals without a first-generation family history of affective disorders recruited among blood donors from the Blood Bank at Rigshospitalet, Copenhagen, as in prior studies.

\section{Diagnostic assessments at inclusion}

The initial diagnostic assessment will be done using the Structured Clinical Interview for DSM-IV-TR Axis I Disorders $^{20}$ categorising patients into bipolar disorder type I or type II as part of daily praxis by the experienced specialists in psychiatry during the patients' 2-year stay in the Copenhagen Affective Disorder Clinic. This clinical diagnosis of bipolar disorder will be confirmed in a semistructured research-based interview using the Schedules for Clinical Assessment in Neuropsychiatry (SCAN) providing an ICD-10 diagnosis. ${ }^{21}$ There will be no attempt to balance the prevalence of bipolar subtypes in the BIO study.

\section{Follow-up}

Besides the assessments at inclusion, patients will be assessed during remitted, depressive and manic/mixed phases. Patients and healthy control individuals will be initially assessed face-to-face and at least every year during the first 4 years and after this, every second year for 5 years.

As part of daily clinical praxis in Copenhagen Affective Disorder Clinic and as part of the BIO-2 substudy, all patients will get access to a smartphone app for electronic continuous monitoring of illness activity during a 5-year follow-up period (see substudy BIO-2). Additionally, research assistants will contact all participants every third month to identify upcoming episodes/onset of illness and to ensure continued participation in the BIO study. At each assessment, the present clinical state (remission, manic, hypomanic, depressive, mixed episode) of all participants will be established according to ICD-10. The severity of depressive and manic symptoms (if present) will be assessed using the 17-item Hamilton Depression Rating Scale (HAMD-17) ${ }^{22}$ and the Young Mania Rating Scale (YMRS) ${ }^{23}$ with a time period of 3 days applied. Remission is defined as score of $\leq 7$ on the HAMD-17 and the YMRS. In this way, upcoming episodes and onset of illness (for healthy individuals) will be assessed with great certainty.

Based on prior findings, ${ }^{2319}$ it is estimated that patients will develop four to five affective episodes on average during the follow-up period, that is, relapse or recurrence, defined as above. It estimated that $20 \%-30 \%$ of the healthy first-generation relatives will develop onset of affective illness compared with $2 \%-5 \%$ among the healthy individuals without a first-generation family history of affective disorders. ${ }^{24}{ }^{25}$ Finally, linkage to Danish nationwide register-based data will be included for all individuals on psychiatric hospitalisations, prescribed medication and socioeconomic variables during the 5-year follow-up.

\section{Investigations}

We will include state-of-the-art methods within clinical assessment as well as biomarkers including a range of new methods:

1. clinical assessments using the Hamilton Depression Scale-17 items (HAMD-17), ${ }^{22}$ the YMRS, ${ }^{23}$ the Functional Assessment Short Test (FAST) (a measure of psychosocial function) ${ }^{26}$;

2. questionnaires including the Hypomania Check list, ${ }^{27}$ Standardized Assessment of Personality-Abbreviated Scale, ${ }^{28}$ the WHO (Five) well-being index ${ }^{29}$ and the Verona Satisfaction Scale-Affective Disorder ${ }^{30}$;

3. standardised fasting blood tests for a large number of potential biomarkers (see the BIO-1, Biological tests section);

4. spot urine samples for oxidative generated damage to DNA and RNA;

5. hair cortisol as a valid and reliable index of long-term systemic cortisol levels ${ }^{31}$;

6. combined heart rate and movement sensor mounted at the thorax (Actiheart ${ }^{32} 33$ );

7. daily electronic smartphone-based self-monitoring of depressive and manic symptoms;

8. daily electronic smartphone-based automatically generated data (eg, data on phone usage, social activity and physical activity);

9. neuropsychological assessment (only during full or partial remission);

10. structural MRI focusing on prefrontal cortex and hippocampus as well as functional MRI (fMRI) (only during full or partial remission).

Clinical assessments will be performed by six $\mathrm{PhD}$ students (master's degree as a Medical Doctor or psychologist). They will be certified in a PhD training course in the SCAN interview and will be trained in using rating scales (HAMD-YMRS, FAST). Inter-rater sessions supervised by senior clinicians from the Copenhagen Affective Disorder Clinic and senior researchers in the BIO study 
Table 1 Overview of the longitudinal assessments during risk periods and following onset of bipolar disorder in the four substudies of the BIO study

\begin{tabular}{|c|c|c|c|c|c|c|c|}
\hline Course & $\begin{array}{l}\text { Healthy first-generation } \\
\text { relatives }\end{array}$ & First episode & Remission & First relapse & Remission & $\begin{array}{l}\text { Second } \\
\text { relapse }\end{array}$ & Others \\
\hline $\mathrm{BIO}-1$ & $x$ & $x$ & $x$ & $x$ & $x$ & $x$ & $x$ \\
\hline $\mathrm{BIO}-3$ & $x$ & & $x$ & & $x$ & & $x$ \\
\hline $\mathrm{BIO}-4$ & $x$ & $x$ & $x$ & $x$ & $x$ & $x$ & $x$ \\
\hline
\end{tabular}

BIO-1: Peripheral blood-based biomarker in bipolar disorder.

BIO-2: Smartphone-based electronic biomarker in bipolar disorder.

$\mathrm{BIO}-3$ : Neurocognitive and brain imaging signatures in bipolar disorder.

$\mathrm{BIO}-4$ : At risk or prodromal phase for bipolar disorder.

$\mathrm{BIO}$, Bipolar Illness Onset study.

will be conducted among the researchers at regular time points during the entire study period.

The BIO study includes four separate but interacting substudies (BIO-1, BIO-2, BIO-3, BIO-4), as illustrated in table 1 with specified aims, background and theoretical basis, and methods, as described in the following. Background and reasons for selection of putative biomarkers is an iterative process presented in each substudy. Nevertheless, as the area of potential biomarkers is constantly evolving and as the substudies cover four different areas, the research protocol does not include systematic reviews of the literature.

\section{BI0-1: peripheral blood-based biomarker in bipolar disorder} Aims

To identify a composite blood-based biomarker that (1) discriminates individuals with bipolar disorder from healthy control individuals, (2) discriminates between manic, depressive and remitted states, (3) predicts emerging affective episodes, and (4) to validate the composite blood-based biomarker against the smartphone-based biomarker, and the neurocognitive and brain imaging signature, and (5) to investigate the change in individual biomarkers as well as the composite bloodbased biomarker following onset of first manic episode, during successive relapses and in the end late stages of the illness. ${ }^{15}$

\section{Background and theoretical basis}

In a series of meta-analyses, we concluded that although a number of candidate peripheral biomarkers related to neuroplasticity, inflammation, oxidative stress and gene expression seem promising, findings are limited by poor study designs, small cross-sectional samples, lack of adjustment for important confounders related to most peripheral biomarkers and poor laboratory methodology. ${ }^{34-37}$ Because of high interindividual variation in peripheral biomarkers, assessment of intraindividual alterations from onset of illness through different affective phases and into the late illness stage is necessary to identify clinically relevant and valid biomarkers, necessitating a longitudinal study design..$^{35} 38$
We have in two longitudinal studies with repeated assessments of patients with bipolar disorder during affective states (manic/mixed, depressive and euthymic) and healthy control individuals found that brain-derived neurotrophic factor (BDNF), ${ }^{39}$ increased oxidative DNA and RNA damage ${ }^{4041}$ and decreased mRNA expression of the PTGDS gene encoding the prostaglandin D synthase enzyme $^{42}$ are markers related to the illness trait in bipolar disorder. The level of the cytokines IL-6 and IL-18 (interleukin) was related to manic episodes only and the activity of GSK-3beta varied with affective states, ${ }^{43}$ suggesting that these may be state markers. ${ }^{44}$ These results have contributed to the research area of biomarkers in bipolar disorder, moving the area closer towards identifying clinically applicable biomarkers.

Nevertheless, it is unlikely that one single biomarker will provide a useful diagnostic tool; instead, a composite of several relevant biomarkers appears as more viable approach. ${ }^{45}$ Recently, promising results have been presented regarding a diagnostic test in unipolar depression comprising serum levels of nine individual biomarkers in peripheral blood. ${ }^{13}$ Similarly, preliminary studies have suggested composite biomarkers for bipolar disorder. ${ }^{46-48}$ We identified a composite biomarker consisting of gene expression from 19 candidate genes for bipolar disorder that accurately discriminated patients with bipolar disorder from healthy control individuals. ${ }^{46}$ Thus, such approaches highly increase the chances of obtaining a high specificity and sensitivity of the composite blood-based biomarker. ${ }^{46-48}$

In order to establish relevant markers of risk and markers related to illness stage, it is necessary to include assessment before onset of illness and during first and recurrent relapses and in the late stages of the illness according to the staging model of bipolar disorder. ${ }^{49}$ Further, the study of early-onset individuals is necessary to evaluate biomarker levels without influence from medication, which may otherwise limit the validity of identified biomarkers.

\section{Methods}

BIO-1 will include repeated clinical assessments and corresponding samples of blood and other tissues among 
the 300 newly diagnosed/first-episode patients with bipolar disorder, the 200 first-generation relatives and the 100 healthy individuals without a first-generation family history of affective disorder.

We will estimate a composite blood-based biomarker based on a number of individual markers including BDNF, neutrophin-3, five different cytokines, gene expression, additional 30 candidate genes and other potential biomarkers (see the Biological tests section), and identify the composite biomarker that correlates best with affective states and Hamilton Depression Rating Scale and YMRS scores of depression and mania, respectively. A final blood-based biomarker will be chosen based on its ability to (1) discriminate patients with bipolar disorder from healthy control individuals and to (2) discriminate between manic, depressive and remitted states in bipolar disorder.

\section{Laboratory procedures}

We will obtain careful standardisation of blood sampling and laboratory analysis by obtaining blood samples in a fasting state and in a 1-hour interval in the morning. At the same day as blood sampling, smoking status, alcohol use, body mass index, menstrual cycle, and so on will be assessed. Blood sampling and all phases of laboratory processing for plasma and DNA/RNA analyses will be done at the Department of Clinical Biochemistry, Rigshospitalet, using standard operational methods conducted by a team of technicians blinded with respect to participant status. All plasma samples will be stored at $-80^{\circ} \mathrm{C}$. The BIO study will include a total of 2400 blood samples: 300 patients $\times 5+200$ healthy first-generation relatives $\times 3+100$ healthy participants without a first-generation family history $\times 3$.

\section{Biological tests}

We will use a multianalyte panel including a large number of potential biomarkers such as plasma levels of Neutrophins3, GSK-3, ß-amyloid Aß40 and Aß42, BDNF, inflammatory markers, high-sensitive C-reactive protein, lipoproteins (very-low-density lipoprotein, low-density lipoprotein, high-density lipoprotein) and specific apolipoproteins (eg, apoE, apoA-I, apoA-II and apoM) as potential markers of low-grade inflammation particularly salient in the early stages of bipolar disorder. Total RNA, microRNA, genomic DNA and histones are isolated from peripheral blood mononuclear cells. Gene expression and alternative slicing of RNA transcripts are analysed using array real-time reverse transcriptase quantitative PCR and next-generation sequencing. Epigenetic modifications of the DNA (eg, methylation) are measured using antibody-based methods or bisulphite treatments in combination with next-generation sequencing. The genomic positions of histones with specific modifications are detected using chromatin immunoprecipitation sequencing. The degree of histone modifications is measured by semiquantitative antibody-based detection.
Measurements of DNA and RNA damage by oxidation are obtained from spot urine samples and analysed using ultra performance liquid chromatography and mass spectrometry.

Hair cortisol will be included as a valid and reliable index of long-term systemic cortisol levels. ${ }^{31}$

We will report on these individual biological tests including comparing patients, first-degree relatives and healthy control individuals, when appropriate. The BIO study sample of 600 participants is rather small for genetic analyses discriminating patients with bipolar disorder from healthy controls but some genetic analyses, including the CACNA1C gene, can be conducted in cooperation with national and international genetic network groups.

\section{Statistical analyses}

Data represent repeated measures within and between individuals and will be analysed using a combination of generalised linear mixed models, integrated data analysis and penalised regression approaches to facilitate the combined feature selection and prediction of the available high-dimensional data. Integrative data analysis ensures that we are able to identify an improved composite bloodbased biomarker since data from different molecular levels are combined in a simultaneous analysis that closely resembles the biological system. ${ }^{50}{ }^{51}$ Further, we will use cross validation or alternatively split sample designs in the development and validation of the composite biomarker. Finally, if possible we will evaluate the biomarker(s) in external non-Danish data sets in collaboration with other international researchers.

A general principle that pertains to statistical analyses of all four substudies is the 'intention to treat' principle. Accordingly, participants who during follow-up get a diagnosis with a higher diagnostic validity than bipolar disorder (ie, a lower ICD-10 diagnostic number, DF00, DF10 and DF20) that may substantially influence the biomarker measures are included in the analyses until onset of symptoms from the disorder but excluded from subsequent analyses. These disorders include significant neurological disorders such as dementia, stroke, brain tumour, multiple sclerosis, Parkinson's disease as well as disorders due to significant psychoactive substance use and schizophrenia.

Furthermore, in all four substudies, the problem of missing data will be alleviated by the use of mixed effect model (for longitudinal measurements) and multiple imputations using chained equations when applicable. If possible, joint modelling will be considered, depending on the missing mechanism observed.

\section{Statistical power}

The study has a power of $80 \%$ to detect a minimum increment of 6.5 percentage points in sensitivity if we assume that the existing diagnostic tools have a sensitivity of $70 \%$ to diagnose bipolar disorder for a patient who has the disorder (see ref 52). Thus, if the composite 
biomarker score increases the sensitivity by a minimum of 6.5 percentage points then we have a power of $80 \%$ to detect that increase based on 300 patients with bipolar disorder using a one-sided exact binomial test (for fixed specificity).

\section{BI0-2: smartphone-based electronic biomarker in bipolar disorder \\ Aims}

To identify a composite smartphone-based electronic biomarker that (1) discriminates patients with bipolar disorder from healthy control individuals, (2) discriminates between manic, depressive and remitted states, (3) predicts emerging affective episodes, and (4) to investigate the change in the composite smartphone-based biomarker following onset of first manic episode, during successive relapses and in the end stages of the illness as according to the staging system by Berk et al. ${ }^{15}$

\section{Background and theoretical basis}

Recently, electronic self-monitoring of the severity of depressive and manic symptoms using text messages has been suggested as an easy and inexpensive way to identify early signs of affective episodes, providing opportunities for mental healthcare providers to intervene shortly after prodromal symptoms first appear. ${ }^{53}$ We have in the MONARCA project developed and tested a smartphone-based electronic monitoring system including daily subjective self-assessments of illness activity in bipolar disorder as well as a bidirectional feedback loop between the patient and clinicians (the MONARCA system ${ }^{54-58}$ ). Using the MONARCA system, fine-grained electronic data were collected during everyday life in naturalistic settings in patients with bipolar disorder. The MONARCA system was reported highly useable and useful by patients with bipolar disorder with a high self-assessment adherence $(87 \%-$ $95 \%$ ), and the patients reported that the MONARCA system helped them to better manage their disease.$^{5455}$ Further, the severity of depressive and manic symptoms was found to correlate with automatically generated smartphone data including (1) physical activity as reflected by the number of changes in cell tower ID per day, ${ }^{59}$ (2) social activity as reflected by the number of incoming and outgoing calls per day, the duration of incoming and outgoing calls per day and the number of outgoing text messages per day, ${ }^{60}$ and (3) voice features collected during phone calls. ${ }^{61}$ Although these findings are encouraging there is a need to integrate self-monitored smartphone data with automatically generated smartphone data on social and physical activities, speech and sleep into one composite smartphone-generated electronic biomarker measure. This composite measure should be modelled to (1) discriminate patients with bipolar disorder from healthy control individuals, (2) have a high correlation with depressive and manic symptoms, (3) discriminate between euthymic, manic and depressive states and (4) early predict emerging affective episodes for the individual patient to increase the possibility for early intervention.
To validate smartphone-based measures of physical activity and sleep, a subset of patients will wear a combined heart rate and movement sensor mounted at the thorax that has been shown to correlate with mood symptoms and affective states and differentiate between patients and controls (Actiheart ${ }^{32}{ }^{33}$ ) like other wearable actigraphs. ${ }^{62-64}$

\section{Methods}

All newly diagnosed/first-episode patients will have access to a smartphone-based system (Monsenso that is developed from the MONARCA system) for continuous self-monitoring as well as fine-grained automatically monitoring of behavioural activity and early identification of emerging affective episodes during the first 2 years and following relapse of episodes during a 5-year follow-up period.

\section{Data analyses}

In contrast to data in BIO-1, data in BIO-2 represent big data collected on a daily basis within individuals. We will use hierarchical Bayesian predictive models that can handle big data through sampling and visualisation techniques that summarise data.

\section{BI0-3: neurocognitive and brain imaging signatures in bipolar disorder \\ Aims}

(1) To identify an integrated brain-based biomarker of bipolar disorder including neurocognitive and neuroimaging measures tapping into 'hot' (ie, emotion laden) and 'cold' (non-emotional) cognition, (2) to examine whether the degree of abnormality in these measures predicts illness onset in the high-risk group and/or relapse in the patient group, (3) to identify developmental trajectories in 'hot' and 'cold' cognitive dysfunction and to identify structural MRI and fMRI correlates in bipolar disorder via longitudinal assessments of high-risk individuals to remission after onset of first manic episode and following successive relapses, and (4) to identify associations between aberrant 'hot' and 'cold' neurocognitive functions, structural and functional brain changes and the composite blood-based and smartphone-based biomarkers. Such 'integrated systems approach' involving identification of patterns of biomarkers (biosignatures) across these multiple levels of investigation is considered imperative for deeper understanding of the dimensions of underlying pathophysiological processes in bipolar disorder. $^{12}$

\section{Background and theoretical basis}

Results from a number of meta-analyses of a large number of cross-sectional studies of patients with bipolar disorder in remission suggest trait-related 'cold' cognitive deficits in attention/processing speed, memory and executive function compared with healthy controls ${ }^{65-68}$ that correlate with everyday functioning. ${ }^{69}$ Cross-sectional comparison of patients at different illness stages revealed more pronounced cognitive deficits during late 
stage compared with early stage in line with the staging hypothesis of bipolar disorder. ${ }^{70}$ However, there are only a few longitudinal studies of neuropsychological functioning with small sample sizes (12 studies including a total of 152 patients with bipolar disorder ${ }^{71}$ ). A meta-analysis of these studies found no support for a progressive nature of cognitive deficits. ${ }^{71}$ However, results from these studies are hampered by a number of limitations including small sample sizes, short follow-up (mean follow-up period of 4.6 years) and high attrition rates (up to $45 \%) .{ }^{71}$ Consequently, it is unclear whether cognitive function assessed with neuropsychological tests deteriorates with the number and duration of illness episodes in bipolar disorder although epidemiological studies consistently revealed increased increasing risk of dementia with the number of episodes ${ }^{7}$ (see also ref 9). Risk of developing dementia long-term ${ }^{6872-74}$ and there are is some evidence for

Deficits in 'hot' cognition are closely linked to emotional disturbances ${ }^{75}$ and difficulties in socioemotional behaviour and interpersonal relations in bipolar disorder. ${ }^{76}$ 'Hot' cognition abnormalities in bipolar disorder have been observed within three domains: (1) emotional processing, (2) reward processing and (3) emotion regulation (reviews in refs 77 78).

Results from a large number of cross-sectional structural imaging studies suggest that patients also show increased lateral ventricular volumes and greater prevalence of white matter hyperintensities. ${ }^{79}$ While these findings are rather unspecific, studies also suggest that treatment with lithium increases the grey matter volume of prefrontal cortex, amygdala and hippocampus. ${ }^{79}$ In addition, a number of functional imaging studies suggest that bipolar disorder is associated with abnormalities within fronto-limbic-subcortical structures. $^{38}$

As long-term, integrative studies are lacking, it is unclear how neurocognitive and brain imaging abnormalities correlate with the staging of bipolar disorder, illness progression and treatment ${ }^{88} 80$ or with changes in biological markers such as neurotropic, inflammatory and oxidative stress markers. ${ }^{81} 82$

\section{Methods}

Using a comprehensive neurocognitive test battery, we will assess all participants from the three groups (patients with bipolar disorder, first-generation relatives and healthy individuals without a family history of affective disorders). Patients will be followed from first onset of affective disorder and during successive periods of remission or at an annual basis (patients with bipolar disorder with no relapse, first-generation relatives with no onset and healthy controls). Among these, a subgroup of 60 patients, 60 healthy relatives (ultra risk) and 30 healthy individuals without a family history of affective disorders (healthy controls) will undergo functional and structural MRI at these time points.

\section{Neurocognitive testing}

Within 'cold' cognition verbal learning/memory and executive function have been highlighted as the most suitable candidates for biomarkers of bipolar disorder. ${ }^{83} 84$ 'Cold' cognition will therefore be assessed with neurocognitive tests probing verbal memory, attention and executive function including the Rey Auditory Verbal Learning Test ${ }^{8586}$ and the WAIS-III letter-number sequencing, RBANS digit span, n-back working memory, verbal fluency and Trail Making Test B. Verbal intelligence will be estimated with the Danish Adult Reading Test.

'Hot' cognition will be assessed with a comprehensive battery of computerised neurocognitive tests outside the scanner probing (1) emotional processing, (2) reward processing and (3) emotion regulation. These include the facial expression recognition and faces using dot-probe tasks from the Emotional Test Battery (P1Vital, Oxford), and an ecologically valid social scenarios test developed by our group. ${ }^{87}$ During fMRI we will also administer the following experimental paradigms: (1) an emotional face processing task using face stimuli from the Nimstim (http://www.macbrain.org/resources.htm), (2) a monetary reward processing task ${ }^{88}$ and (3) a negative affective picture task using validated stimuli sets from the International Affective Picture System developed in collaboration with researchers at the University of Chicago. In addition, we will explore the neuronal basis for 'cold' cognition (executive function) using n-back working memory and picture encoding tasks programmed in-house ${ }^{89}$ Finally, self-report measures (BIS/BAS, and the CERQ ${ }^{90}$ ) are used to assess reward responsiveness and habitual emotional regulation strategies.

\section{Structural MRI and fMRI}

\section{Structural MR}

Using T1-weighted images acquired at a 3T Siemens scanner at the Copenhagen University Hospital, Rigshospitalet, we will focus on lateral ventricular volumes, grey matter volume of prefrontal cortex, amygdala and hippocampus, relative to whole brain volume. Specifically, segmentation and analysis of subcortical and regional cortical volume, shape and grey matter density will be conducted with FMRIB Software Library (FSL) tools, including the FMRIB's Integrated Registration and Segmentation Tool, the FSL-VBM tool and FSL vertex (shape) analysis (http://fsl.fmrib.ox.ac.uk/).

\section{Functional MRI}

T2-weighted images will be acquired to investigate white matter hyperintensities. We will also use fMRI to investigate neuronal underpinnings of 'hot' and 'cold' cognition with the previously described experimental paradigms. fMRI data processing will be carried out with the FMRI Expert Analysis Tool, part of FMRIB's Software Library (www.fmrib.ox.ac.uk/fsl). We will examine mean per cent BOLD signal change within predefined hippocampal and amygdala regions of interest obtained in standard space 
with mri3dX (http://www.idoimaging.com/program/ 160). In addition, whole-brain exploratory analysis will be conducted to explore neural activity differences in other cortical regions. For this group analysis, individual contrasts of interest will be included in separate general linear models with non-parametric permutation inference $(\mathrm{n}=5000)$ using the 'randomize' algorithm implemented in FSL. ${ }^{91}$

\section{Statistical power}

The above sample size for participants undergoing fMRI assessments is determined based on our previous fMRI studies. In particular, inclusion of about 17-22 participants per treatment/diagnostic group (matched for age and gender) had a power of $>0.8$ to detect differences between groups in neural and cognitive response to emotional faces (eg, refs 9293 and Miskowiak et al, under review) at an alpha level of $\mathrm{p}<0.05$ for cross-sectional designs. For longitudinal designs, we were able to demonstrate differences between groups in the change in task-related neural activity and in hippocampal structure with a sample of about 40 participants per group. ${ }^{94-96}$ Given this, our inclusion of 60 participants per group is expected to ensure adequate statistical power for both the cross-sectional and longitudinal parts of the fMRI study.

\section{BI0-4: at risk or prodromal phase for bipolar disorder Aims}

To test whether (1) the composite blood-based biomarker, (2) the composite electronic smartphone-based biomarker and (3) the neurocognitive signature for bipolar disorder predict onset of illness (depression or mania) among a healthy (ie, non-syndromal level) highrisk population of first-degree siblings and offspring to the patients with newly diagnosed/first-episode mania/ bipolar disorder, included in BIO-1, BIO-2 and BIO-3.

\section{Background and theoretical basis}

First-generation relatives to patients with bipolar disorder have a ninefold increased risk of developing bipolar disorder and a twofold to threefold increased risk of developing unipolar disorder. ${ }^{1}$ Although there is a great need for early detection and primary prevention of onset of illness among relatives to patients with bipolar disorder, recent prior attempts have not been successful due to retrospective designs, poor characterisation of high-risk individuals and small sample sizes. ${ }^{97}$ This is the first time a composite blood-based, a composite smartphone-based and a composite neurocognitive biomarker identified among patients with bipolar disorder will be investigated among their healthy relatives as a predictor measure of onset of illness. ${ }^{18}$ This approach increases the chances of obtaining a high specificity and sensitivity of the composite biomarkers.

\section{Methods}

This BIO-4 includes first-generation relatives (siblings and offspring aged 15-40 years) to the recruited patients with first manic or bipolar diagnosis. All recruited patients will be asked about the lifetime psychiatric history of first-degree relatives (their biological parents, siblings and offspring) based on the Brief Screening for Family Psychiatric History questionnaire described by Weissman and colleagues. ${ }^{98}$ We expect that at least 200 first-generation relatives will be asymptomatic or present mild symptoms or prodromal patterns to affective disorders and will be included in the study. Biological tissues will be drawn (as part of BIO-1) and neurocognitive function will be assessed on all individuals and brain imaging will be done on 60 individuals with longitudinal assessments.

\section{Statistical analyses}

BIO-4 will use a combination of penalised regression techniques, and random forests to infer the importance of the original and combined markers and to compare the similarity of prediction error from the models with and without combining the markers.

\section{Feasibility}

The BIO study is fully feasible as patients are recruited as part of daily healthcare for patients referred to the Copenhagen Affective Disorder Clinic.

\section{Ethical considerations}

The BIO study has been approved by the Local Ethical Committee (H-7-2014-007) and the data agency, Capital Region of Copenhagen (RHP-2015-023). According to these specifications, adolescents aged 15-18 years will be invited only if parents have given consent. Data will be saved, encrypted and assessed according to the regulations from the Capital Region of Denmark. The study complies with the Declaration of Helsinki principles (Seoul, October 2008).

\section{Dissemination}

Study results will be presented in peer-reviewed journals and at international conferences in accordance with relevant reporting guidelines. ${ }^{99} 100$

\section{DISCUSSION}

\section{Summary}

It is expected that the $\mathrm{BIO}$ cohort will provide valid biological, electronic, neurocognitive and neuroimaging data and for the first time longitudinally identify changes in biomarkers during different stages of bipolar illness, that is, at risk stage, following onset, during first relapse and recurrent relapses and in the late stages of the illness as according to Berk et al. ${ }^{15}$

\section{Limitations}

Some limitations of the BIO study should be noted beforehand. First, the rather extensive initial assessment study procedure may potentially result in selection of participants who are intrinsically positive towards clinical research and readily willing to cooperate. Nevertheless, we expect that such selection will be decreased as it is likely that the vast majority of more than 100 newly diagnosed/ 
first-episode patients with bipolar disorder referred to the Copenhagen Affective Disorder Clinic each year will accept participation in the BIO study as this implies an extensive clinical evaluation.

Second, attrition may increase during long-term follow-up and patients who stay in the study may adhere more to treatment in general. Such selection is inherent in clinical longitudinal research, and the large number of participants that will be included will increase external validity. Third, potential confounding effects of psychotropic medication may influence comparisons between patients with bipolar disorder and healthy control individuals as well as comparisons within patients as the vast majority of patients will get medication that may change during the course of illness. Lithium, mood stabilisers and antipsychotics may have effects on the collected biological, smartphone-based, neuropsychological and brain imaging data. Effects of medication on biological measures are not clear ${ }^{101}$ although analyses from systematic reviews and meta-analyses involving only patients with bipolar disorder have not found clear effects of medication on cytokines, ${ }^{34}{ }^{35} \mathrm{BDNF}^{37}$ and gene expression $^{36}$ nor have subsequent individual studies on cytokines, ${ }^{44} 102$ BDNF, ${ }^{39} 102$ gene expression ${ }^{46}$ or DNA and RNA damage. ${ }^{40}$ Effects of medication on electronic smartphone-generated data as well as on neuropsychological and brain imaging data are poorly investigated and warrant further studies. ${ }^{103}$

Fourth, due to the large number of biological and statistical tests included in the BIO study, chance findings may occur in relation to the individual biological test. However, the aim of the BIO study is to identify a composite biomarker measure related to bipolar illness, depression and mania using cross validation or alternatively split sample designs in the development and validation of the composite biomarker.

Finally, the BIO study does not include (randomised) interventions limiting causal interpretations of the results. Nevertheless, with the BIO prospective, repeated measures design it is possible to identify valid associations between the composite measures (of biological, electronic, neuropsychological and brain imaging data) and depressive and manic symptoms and states.

\section{Strengths}

The BIO study is the first study aiming to identify (1) a composite blood-based biomarker, (2) a composite electronic smartphone-based biomarker and (3) a neurocognitive signature for bipolar disorder as well as to measure the same biomarkers in newly diagnosed/ first-episode patients with bipolar disorder and their healthy first-generation relatives. It is possible to recruit newly diagnosed/first-episode patients with mania/ bipolar disorder as all such patients from the entire Capital Region of Denmark are referred to the Copenhagen Affective Disorder Clinic and routinely asked for inclusion in the BIO study. Long-term attrition is supposed to be low as all patients will be followed by the Copenhagen Affective Disorder Clinic for the first 2 years and subsequently in other treatment settings in the Capital Region of Denmark. Including longitudinal assessment of healthy individuals is of paramount importance to control for the effect of timing and ageing, ${ }^{104}$ but among all studies on biomarkers in bipolar disorder, this has been done only in two studies from our group. ${ }^{44}$ The study will be performed by an experienced international research group, combining expertise from all areas of the study.

\section{CONCLUSION}

The BIO study is a large long-term cohort study on biomarkers in bipolar disorder and we expect that the findings for the first time will be representative of biomarkers in bipolar disorder in general as no prior study on newly diagnosed/first-episode bipolar disorder has been conducted. It is expected that the BIO cohort will provide valid biological, electronic, neuropsychological and brain imaging longitudinal data.

\section{Author affiliations}

${ }^{1}$ Department of Psychiatry, Psychiatric Center Copenhagen, Copenhagen, Denmark ${ }^{2}$ Institute of Clinical Medicine, University of Copenhagen, Copenhagen, Denmark ${ }^{3}$ Department of Clinical Biochemistry, Rigshospitalet, Copenhagen, Denmark ${ }^{4}$ Department of Biostatistics, University of Copenhagen, Copenhagen, Denmark ${ }^{5}$ Department of Applied Mathematics and Computer Science, Technical University of Denmark, Kongens Lyngby, Denmark

${ }^{6}$ Gene Regulation Bioinformatics at the Bioinformatics Centre, Department of Biology/BRIC, University of Copenhagen, Copenhagen, Denmark

${ }^{7}$ The Centre of Inflammation and Metabolism at Department of Infectious Diseases, Rigshospitalet, Copenhagen, Denmark

${ }^{8}$ Laboratory of Clinical Pharmacology, Rigshospitalet, Copenhagen, Denmark ${ }^{9}$ Department of Psychiatry and Pharmacology, University of Toronto, Brain and Cognition Discovery Foundation, Toronto, Ontario, Canada

${ }^{10}$ McMaster University, Hamilton, Ontario, Canada

${ }^{11}$ Department and Institute of Psychiatry, and Laboratory of Neuroscience (LIM27), University of São Paulo Medical School, São Paulo, Brazil

${ }^{12}$ IT University Copenhagen, Copenhagen, Denmark

${ }^{13}$ Create-Net: Center for Research and Telecommunications Experimentation for Networked Communities, Trento, Italy

${ }^{14}$ Neurobiology Research Unit and Center for Integrated Molecular Brain Imaging, Rigshospitalet, Copenhagen, Denmark

${ }^{15}$ Department of Psychiatry, University of Pittsburgh, Western Psychiatric Institute and Clinic, Pittsburgh, Pennsylvania, USA

Correction notice This paper has been amended since it was published Online First. Owing to a scripting error, some of the publisher names in the references were replaced with 'BMJ Publishing Group'. This only affected the full text version, not the PDF. We have since corrected these errors and the correct publishers have been inserted into the references.

Acknowledgements The validity of the research cannot be influenced by any of these potential secondary interests (such as financial gain or personal relationship).

Contributors LVK designed the study together with MV, KM, KWM and MFJ. LVK drafted the study protocol and the manuscript. All authors contributed to development of the study protocol and to editing the manuscript and read and approved the final version. KM, LBN, RFS, CE, BKP, HEP, RSM, FK, WFG and MV contributed specifically to BI0-1 on peripheral blood-based biomarkers. MFJ, OW, $\mathrm{JB}, \mathrm{MF}$ and $\mathrm{OM}$ contributed specifically to BIO-2 on smartphone-based electronic biomarkers. KWM, GMK and MP contributed specifically to BIO-3 on neurocognitive and brain imaging signatures. MV contributed specifically to BIO-4 on at risk or prodromal phase of bipolar disorder (in addition to BI0).

Funding The study is funded by grants from the Mental Health Services, Capital Region of Denmark, TheDanish Council for Independent Research, Medical 
Sciences (DFF-4183-00570), Weimans Fund, Markedsmodningsfonden (the Market Development Fund 2015-310), Gangstedfonden (A29594), Helsefonden (16-B-0063), Innovation Fund Denmark (the Innovation Fund, Denmark, 516400001B), Copenhagen Center for Health Technology (CACHET), EU H2020 ITN (EU project 722561), Augustinusfonden (16-0083), Lundbeck Foundation (R2152015-4121).

Competing interests LVK has within the preceding three years been a consultant for Lundbeck, AstraZeneca and Sunovion. KWM has received consultancy fees in the past three years from Lundbeck and Allergan. MFJ has been a consultant for Eli-Lilly and Lundbeck. MV has within the preceding three years been a consultant for AstraZeneca and Servier. FK has been a speaker for Ache, Daiichi Sankyoand Janssen. MP is a consultant for Roche Pharmaceuticals. RSM: Advisory boards: Lundbeck, Pfizer, AstraZeneca, Eli-Lilly, Janssen, Ortho Purdue, Johnson \& Johnson, Moksha8, Sunovion, Mitsubishi, Takeda, Forest, Otsuka, Bristol-Myers Squibb, Shire. Speaker fees: Lundbeck, Pfizer, AstraZeneca, Eli-Lilly, Janssen Ortho, Purdue, Johnson \& Johnson, Moksha8, Sunovion, Mitsubishi, Takeda, Forest, Otsuka, Bristol-Myers Squibb, Shire. Research grants: Lundbeck, Janssen Ortho, Shire, Purdue, AstraZeneca, Pfizer, Otsuka, Allergan. HEP has received a research grant from Boehringer Ingelheim. GMK has received honoraria as Field Editor of the International Journal of Neuropsychopharmacology and as scientific advisor for $\mathrm{H}$ Lundbeck A/S. JB and MF are co-founders and shareholders in Monsenso. LBN, RFS and WFG declare no competing interests. The validity of the research cannot be influenced by any of these potential secondary interests (such as financial gain or personal relationship).

Patient consent The informed consent form from the Local Ethical Committee in Copenhagen is signed by the participants and the researcher.

Ethics approval The study has been approved by the Local Ethical Committee (H-72014-007) and the data agency, Capital Region of Copenhagen (RHP-2015-023).

Provenance and peer review Not commissioned; externally peer reviewed.

Open Access This is an Open Access article distributed in accordance with the Creative Commons Attribution Non Commercial (CC BY-NC 4.0) license, which permits others to distribute, remix, adapt, build upon this work non-commercially, and license their derivative works on different terms, provided the original work is properly cited and the use is non-commercial. See: http://creativecommons.org/ licenses/by-nc/4.0/

(c) Article author(s) (or their employer(s) unless otherwise stated in the text of the article) 2017. All rights reserved. No commercial use is permitted unless otherwise expressly granted.

\section{REFERENCES}

1. Lohoff FW, Berettini WH. Genetics of bipolar disorder. In: Yatham LN, Maj M, eds. Bipolar disorder: clinical and neurobiological foundations. Singapore: wiley-blackwell, 2010:110-23.

2. Kessing LV, Hansen MG, Andersen PK, et al. The predictive effect of episodes on the risk of recurrence in depressive and bipolar disorders - a life-long perspective. Acta Psychiatr Scand 2004;109:339-44.

3. Kessing LV, Hansen MG, Andersen PK. Course of illness in depressive and bipolar disorders. naturalistic study, 1994-1999. Br J Psychiatry 2004;185:372-7.

4. Post RM, Fleming J, Kapczinski F. Neurobiological correlates of illness progression in the recurrent affective disorders. J Psychiatr Res 2012;46:561-73.

5. Schneider MR, DelBello MP, McNamara RK, et al. Neuroprogression in bipolar disorder. Bipolar Disord 2012;14:356-74.

6. Kessing LV, Nilsson FM. Increased risk of developing dementia in patients with Major affective disorders compared to patients with other medical illnesses. J Affect Disord 2003;73:261-9.

7. Kessing LV, Andersen PK. Does the risk of developing dementia increase with the number of episodes in patients with depressive disorder and in patients with bipolar disorder? J Neurol Neurosurg Psychiatry 2004;75:1662-6.

8. da Silva J, Gonçalves-Pereira M, Xavier M, et al. Affective disorders and risk of developing dementia: systematic review. $\mathrm{Br} J$ Psychiatry 2013;202:177-86.

9. Kessing LV, Andersen PK. Evidence for clinical progression of unipolar and bipolar disorders. Acta Psychiatr Scand 2017;135:51-64. in press.

10. Kessing LV. Diagnostic stability in bipolar disorder in clinical practise as according to ICD-10. J Affect Disord 2005;85:293-9.
11. Baldessarini RJ, Tondo L, Baethge CJ, et al. Effects of treatment latency on response to maintenance treatment in manic-depressive disorders. Bipolar Disord 2007;9:386-93.

12. Phillips ML, Kupfer DJ. Bipolar disorder diagnosis: challenges and future directions. Lancet 2013;381:1663-71.

13. Papakostas GI, Shelton RC, Kinrys G, et al. Assessment of a multi-assay, serum-based biological diagnostic test for major depressive disorder: a pilot and replication study. Mol Psychiatry 2013;18:332-9.

14. Fries GR, Pfaffenseller B, Stertz L, et al. Staging and neuroprogression in bipolar disorder. Curr Psychiatry Rep 2012;14:667-75.

15. Berk M, Berk L, Dodd S, et al. Stage managing bipolar disorder. Bipolar Disord 2014;16:471-7.

16. Mclntyre RS, Correll C. Predicting and preventing bipolar disorder: the need to fundamentally advance the strategic approach. Bipolar Disord 2014;16:451-4. in press.

17. Grande I, Magalhães PV, Chendo I, et al. Staging bipolar disorder: clinical, biochemical, and functional correlates. Acta Psychiatr Scand 2014;129:437-44.

18. Kapczinski F, Magalhães PV, Balanzá-Martinez V, et al. Staging systems in bipolar disorder: an International Society for Bipolar Disorders Task Force Report. Acta Psychiatr Scand 2014;130:354-63.

19. Kessing LV, Hansen HV, Hvenegaard A, et al. Treatment in a specialised out-patient mood disorder clinic v. standard out-patient treatment in the early course of bipolar disorder: randomised clinical trial. Br J Psychiatry 2013;202:212-9.

20. First MB, Spitzer M, Gibbon M, et al. Structured clinical interview for DSM-IV-TR Axis I Disorders. New York: Biometrics Research Department, New York State Psychiatric Institute, New York, 2002.

21. Wing JK, Babor T, Brugha T, et al. SCAN. Schedules for Clinical Assessment in Neuropsychiatry. Arch Gen Psychiatry 1990;47:589-93.

22. Hamilton M. A rating scale for depression. J Neurol Neurosurg Psychiatry 1960;23:56-62.

23. Young RC, Biggs JT, Ziegler VE, et al. A rating scale for mania: reliability, validity and sensitivity. Br J Psychiatry 1978;133:429-35.

24. Vinberg M, Miskowiak K, Kessing LV. Risk markers for affective disorder, a seven-years follow up study of a twin cohort at low and high risk for affective disorder. $J$ Psychiatr Res 2013;47:565-71.

25. Hillegers $\mathrm{MH}$, Reichart CG, Wals $\mathrm{M}$, et al. Five-year prospective outcome of psychopathology in the adolescent offspring of bipolar parents. Bipolar Disord 2005;7:344-50.

26. Rosa AR, Sánchez-Moreno J, Martínez-Aran A, et al. Validity and reliability of the Functioning Assessment Short Test (FAST) in bipolar disorder. Clin Pract Epidemiol Ment Health 2007;3:5.

27. Angst J, Adolfsson R, Benazzi F, et al. The HCL-32: towards a selfassessment tool for hypomanic symptoms in outpatients. J Affect Disord 2005;88:217-33.

28. Bukh JD, Bock C, Vinberg M, et al. Clinical utility of Standardised Assessment of Personality - Abbreviated Scale (SAPAS) among patients with first episode depression. J Affect Disord 2010;127:199-202

29. Bech $\mathrm{P}$, Olsen LR, Kjoller M, et al. Measuring well-being rather than the absence of distress symptoms: a comparison of the SF-36 Mental Health subscale and the WHO-Five Well-Being Scale. Int $J$ Methods Psychiatr Res 2003;12:85-91.

30. Kessing LV, Hansen HV, Ruggeri M, et al. Satisfaction with treatment among patients with depressive and bipolar disorders. Soc Psychiatry Psychiatr Epidemiol 2006;41:148-55.

31. Stalder T, Kirschbaum C. Analysis of cortisol in hair--state of the art and future directions. Brain Behav Immun 2012;26:1019-29.

32. Faurholt-Jepsen M, Brage S, Vinberg M, et al. Differences in psychomotor activity in patients suffering from unipolar and bipolar affective disorder in the remitted or $\mathrm{mild} / \mathrm{moderate}$ depressive state. $J$ Affect Disord 2012;141:457-63.

33. Faurholt-Jepsen M, Brage S, Vinberg M, et al. State-related differences in the level of psychomotor activity in patients with bipolar disorder - Continuous heart rate and movement monitoring. Psychiatry Res 2016;237:166-74.

34. Munkholm K, Vinberg M, Vedel Kessing L. Cytokines in bipolar disorder: a systematic review and meta-analysis. J Affect Disord 2013;144:16-27.

35. Munkholm K, Braüner JV, Kessing LV, et al. Cytokines in bipolar disorder vs. healthy control subjects: a systematic review and metaanalysis. J Psychiatr Res 2013;47:1119-33.

36. Munkholm K, Vinberg M, Berk M, et al. State-related alterations of gene expression in bipolar disorder: a systematic review. Bipolar Disord 2012;14:684-96. 
37. Munkholm K, Vinberg M, Kessing LV. Peripheral blood brain-derived neurotrophic factor in bipolar disorder: a comprehensive systematic review and meta-analysis. Mol Psychiatry 2016;21:216-28.

38. Frey BN, Andreazza AC, Houenou J, et al. Biomarkers in bipolar disorder: a positional paper from the International Society for bipolar Disorders biomarkers Task Force. Aust N Z J Psychiatry 2013;47:321-32.

39. Munkholm K, Pedersen BK, Kessing LV, et al. Elevated levels of plasma brain derived neurotrophic factor in rapid cycling bipola disorder patients. Psychoneuroendocrinology 2014;47:199-211.

40. Jacoby AS, Vinberg M, Poulsen HE, et al. Increased DNA and RNA damage by oxidation in patients with bipolar I disorder. Trans/ Psychiatry 2016;6:e867.

41. Munkholm K, Poulsen HE, Kessing LV, et al. Elevated levels of urinary markers of oxidatively generated DNA and RNA damage in bipolar disorder. Bipolar Disord 2015;17:257-68.

42. Munkholm K, Peijs L, Kessing LV, et al. Reduced mRNA expression of PTGDS in peripheral blood mononuclear cells of rapid-cycling bipolar disorder patients compared with healthy control subjects. Int J Neuropsychopharmacol 2014;18:pyu101.

43. Jacoby AS, Munkholm K, Vinberg M, et al. Glycogen synthase kinase-3 $\beta$ in patients with bipolar I disorder: results from a prospective study. Bipolar Disord 2016;18:334-41.

44. Munkholm K, Weikop P, Kessing LV, et al. Elevated levels of IL-6 and IL-18 in manic and hypomanic states in rapid cycling bipolar disorder patients. Brain Behav Immun 2015;43:205-13

45. Goldstein BI, Young LT. Toward clinically applicable biomarkers in bipolar disorder: focus on BDNF, inflammatory markers, and endothelial function. Curr Psychiatry Rep 2013;15:425.

46. Munkholm K, Peijs L, Vinberg M, et al. A composite peripheral blood gene expression measure as a potential diagnostic biomarker in bipolar disorder. Transl Psychiatry 2015;5:e614.

47. Dickerson F, Schroeder J, Stallings C, et al. Multianalyte markers of schizophrenia and bipolar disorder: a preliminary study. Schizophr Res 2015;168:450-5.

48. Haenisch F, Cooper JD, Reif A, et al. Towards a blood-based diagnostic panel for bipolar disorder. Brain Behav Immun 2016;52:49-57.

49. Berk M, Conus P, Lucas N, et al. Setting the stage: from prodrome to treatment resistance in bipolar disorder. Bipolar Disord 2007:9:671-8.

50. Brink-Jensen K, Bak S, Jørgensen K, et al. Integrative analysis of metabolomics and transcriptomics data: a unified model framework to identify underlying system pathways. PLoS One 2013;8:e72116.

51. Hastie T, Tibshirani R, Friedman J. Penalised regression.the elements of Statistical Learning: data Mining, Inference, and prediction. 2nd ed: Spring Verlag, 2009.

52. Phelps J, Ghaemi SN. The mistaken claim of bipolar 'overdiagnosis': solving the false positives problem for DSM-5/ICD11. Acta Psychiatr Scand 2012;126:395-401.

53. Bopp JM, Miklowitz DJ, Goodwin GM, et al. The longitudinal course of bipolar disorder as revealed through weekly text messaging: a feasibility study. Bipolar Disord 2010;12:327-34.

54. Bardram JE, Frost M, Szántó K, et al, Designing mobile health technology for bipolar disorder: a field trial of the MONARCA System. Proceedings of the SIGCHI Conference on Human Factors in Computing Systems. 2013; 2627-36

55. Faurholt-Jepsen M, Frost M, Ritz C, et al. Daily electronic selfmonitoring in bipolar disorder using smartphones - the MONARCA trial: a randomized, placebo-controlled, single-blind, parallel group trial. Psychol Med 2015;45:2691-704.

56. Frost M, Doryab A, Faurholt-Jepsen M, et al. Supportingdisease insight through data analysis: refinements of the monarca selfassessment system. Proceedings of the 2013 ACM international joint conference on Pervasive andubiquitous computing. New York, NY, USA: ACM Press, 20143.

57. Bardram JE, Frost M, Szántó, K, et al. The MONARCA selfassessment system: a persuasive personal monitoring system for bipolar patients. Proceedings of the 2nd ACM SIGHIT International Health Informatics Symposium (IHI '12). New York, NY, USA: ACM, 2012:21-30.

58. Gravenhorst F, Muaremi A, Bardram J, et al. Mobile phones as medical devices in mental disorder treatment: an overview. Pers Ubiquitous Comput 2015;19:335-53.

59. Faurholt-Jepsen $M$, Frost $M$, Vinberg $M$, et al. Smartphone data as objective measures of bipolar disorder symptoms. Psychiatry Res 2014;217:124-7.

60. Faurholt-Jepsen M, Vinberg M, Frost M, et al. Smartphone data as an electronic biomarker of illness activity in bipolar disorder. Bipolar Disord 2015;17:715-28.
61. Faurholt-Jepsen M, Busk J, Frost M, et al. Voice analysis as an objective state marker in bipolar disorder. Transl Psychiatry 2016;6:e856.

62. Faedda GL, Ohashi K, Hernandez M, et al. Actigraph measures discriminate pediatric bipolar disorder from attention-deficit/ hyperactivity disorder and typically developing controls. J Child Psychol Psychiatry 2016;57:706-16.

63. Indic P, Salvatore P, Maggini C, et al. Scaling behavior of human locomotor activity amplitude: association with bipolar disorder. PLoS One 2011;6:e20650.

64. Valenza G, Nardelli M, Lanatà A, et al. Wearable monitoring for mood recognition in bipolar disorder based on history-dependent long-term heart rate variability analysis. IEEE J Biomed Health Inform 2014;18:1625-35.

65. Torres IJ, Boudreau VG, Yatham LN. Neuropsychological functioning in euthymic bipolar disorder: a meta-analysis. Acta Psychiatr Scand Supp/ 2007:17-26.

66. Bora E, Yucel M, Pantelis C. Cognitive endophenotypes of bipolar disorder: a meta-analysis of neuropsychological deficits in euthymic patients and their first-degree relatives. J Affect Disord 2009;113:1-20.

67. Mann-Wrobel MC, Carreno JT, Dickinson D. Meta-analysis of neuropsychological functioning in euthymic bipolar disorder: an update and investigation of moderator variables. Bipolar Disord 2011;13:334-42.

68. Bourne C, Aydemir Ö, Balanzá-Martínez V, et al. Neuropsychological testing of cognitive impairment in euthymic bipolar disorder: an individual patient data meta-analysis. Acta Psychiatr Scand 2013;128:149-62.

69. Depp CA, Mausbach BT, Harmell AL, et al. Meta-analysis of the association between cognitive abilities and everyday functioning in bipolar disorder. Bipolar Disord 2012;14:217-26.

70. Rosa AR, Magalhães PV, Czepielewski L, et al. Clinical staging in bipolar disorder: focus on cognition and functioning. J Clin Psychiatry 2014;75:e450-e456.

71. Samamé C, Martino DJ, Strejilevich SA. Longitudinal course of cognitive deficits in bipolar disorder: a meta-analytic study. J Affect Disord 2014;164:130-8.

72. Kessing LV, Olsen EW, Mortensen PB, et al. Dementia in affective disorder: a case-register study. Acta Psychiatr Scand 1999;100:176-85.

73. Wu KY, Chang CM, Liang HY, et al. Increased risk of developing dementia in patients with bipolar disorder: a nested matched casecontrol study. Bipolar Disord 2013;15:787-94.

74. Chen MH, Li CT, Tsai CF, et al. Risk of subsequent dementia among patients with bipolar disorder or Major depression: a nationwide longitudinal study in Taiwan. J Am Med Dir Assoc 2015;16:504-8.

75. Strakowski SM, Adler CM, Holland SK, et al. A preliminary FMRI study of sustained attention in euthymic, unmedicated bipolar disorder. Neuropsychopharmacology 2004;29:1734-40.

76. Kerr N, Dunbar RI, Bentall RP. Theory of mind deficits in bipolar affective disorder. J Affect Disord 2003;73:253-9.

77. Wessa M, Linke J. Emotional processing in bipolar disorder: behavioural and neuroimaging findings. Int Rev Psychiatry 2009;21:357-67.

78. Phillips ML, Swartz HA. A critical appraisal of neuroimaging studies of bipolar disorder: toward a new conceptualization of underlying neural circuitry and a road map for future research. Am J Psychiatry 2014; $171: 829-43$

79. Hallahan B, Newell J, Soares JC, et al. Structural magnetic resonance imaging in bipolar disorder: an international collaborative mega-analysis of individual adult patient data. Biol Psychiatry 2011;69:326-35

80. Mora E, Portella MJ, Forcada I, et al. Persistence of cognitive impairment and its negative impact on psychosocial functioning in lithium-treated, euthymic bipolar patients: a 6-year follow-up study. Psychol Med 2013;43:1187-96.

81. Bauer IE, Pascoe MC, Wollenhaupt-Aguiar B, et al. Inflammatory mediators of cognitive impairment in bipolar disorder. $J$ Psychiatr Res 2014:56:18-27.

82. Lim CS, Baldessarini RJ, Vieta E, et al. Longitudinal neuroimaging and neuropsychological changes in bipolar disorder patients: review of the evidence. Neurosci Biobehav Rev 2013;37:418-35.

83. Balanzá-Martínez V, Rubio C, Selva-Vera G, et al. Neurocognitive endophenotypes (endophenocognitypes) from studies of relatives of bipolar disorder subjects: a systematic review. Neurosci Biobehav Rev 2008;32:1426-38.

84. Raust A, Daban C, Cochet B, et al. Neurocognitive performance as an endophenotype for bipolar disorder. Front Biosci 2014;6:89-103.

85. Rey A. Psychological examination of traumatic encephalopathy. Archieves de Psychologic 1941;28:286-340. 
86. Rey A. L'examen clinique en psychologie Clinical tests in psychology. Paris: Presses Universitaires de France, 1964.

87. Kjærstad HL, Vinberg M, Goldin PR, et al. Impaired down-regulation of negative emotion in self-referent social situations in bipola disorder: a pilot study of a novel experimental paradigm. Psychiatry Res 2016;238:318-25.

88. Chase HW, Nusslock R, Almeida JR, et al. Dissociable patterns of abnormal frontal cortical activation during anticipation of an uncertain reward or loss in bipolar versus major depression. Bipolar Disord 2013;15:839-54.

89. Miskowiak K, O'Sullivan U, Harmer CJ. Erythropoietin enhances hippocampal response during memory retrieval in humans. $J$ Neurosci 2007;27:2788-92.

90. Garnefski N, Kraaij V, Spinhoven P. Negative life events, cognitive emotion regulation and emotional problems. Pers Individ Dif 2001;30:1311-27.

91. Winkler AM, Ridgway GR, Webster MA, et al. Permutation inference for the general linear model. Neuroimage 2014;92:381-97.

92. Miskowiak K, O'Sullivan U, Harmer CJ. Erythropoietin reduces neural and cognitive processing of fear in human models of antidepressant drug action. Biol Psychiatry 2007;62:1244-50.

93. Miskowiak KW, Glerup L, Vestbo C, et al. Different neural and cognitive response to emotional faces in healthy monozygotic twins at risk of depression. Psychol Med 2015;45:1447-58.

94. Miskowiak KW, Vinberg M, Macoveanu J, et al. Effects of erythropoietin on hippocampal volume and memory in mood disorders. Biol Psychiatry 2015;78:270-7.

95. Miskowiak KW, Macoveanu J, Vinberg M, et al. Effects of erythropoietin on memory-relevant neurocircuitry activity and recall in mood disorders. Acta Psychiatr Scand 2016;134:249-59.
96. Miskowiak KW, Vinberg M, Glerup L, et al. Neural correlates of improved executive function following erythropoietin treatment in mood disorders. Psychol Med 2016;46:1679-91.

97. Brietzke E, Mansur RB, Soczynska JK, et al. Towards a multifactorial approach for prediction of bipolar disorder in at risk populations. J Affect Disord 2012;140:82-91.

98. Weissman MM, Wickramaratne P, Adams P, et al. Brief screening for family psychiatric history: the family history screen. Arch Gen Psychiatry 2000;57:675-82.

99. Gallo V, Egger M, McCormack V, et al. Strengthening the Reporting of Observational studies in Epidemiology - Molecular Epidemiology (STROBE-ME): an extension of the STROBE statement. Eur J Clin Invest 2012:42:1-16.

100. von Elm E, Altman DG, Egger M, et al. The Strengthening the Reporting of Observational studies in Epidemiology (STROBE) Statement: guidelines for reporting observational studies. Int J Surg 2014;12:1495-9.

101. van den Ameele S, van Diermen L, Staels W, et al. The effect of mood-stabilizing drugs on cytokine levels in bipolar disorder: a systematic review. J Affect Disord 2016;203:364-73.

102. Jacoby AS, Munkholm K, Vinberg M, et al. Cytokines, brain-derived neurotrophic factor and C-reactive protein in bipolar I disorder Results from a prospective study. J Affect Disord 2016;197:167-74.

103. Laidi C, Houenou J. Brain functional effects of psychopharmacological treatments in bipolar disorder. Eur Neuropsychopharmacol 2016;26:1695-740.

104. Munkholm K, Lenskjold T, Jacoby AS, et al. Glycogen synthase Kinase- $3 \beta$ : variation over time and the possible association with mood and cognition in healthy individuals. Neuropsychobiology 2016;73:108-15. 